\title{
Base functions effect in stability finite element analysis of compressed plate
}

\author{
Ivana Veghova ${ }^{1, *}$ and Martin Psotny ${ }^{1}$ \\ ${ }^{1}$ Slovak University of Technology, Faculty of Civil Engineering, Department of Structural \\ Mechanics, Radlinskeho 11, Bratislava 810 05, Slovakia
}

\begin{abstract}
Geometric nonlinear solution of a compressed plate is presented in this paper. Basic assumptions are specified and incremental conditional equations are derived from the variational principle of minimum of total potential energy. Full Newton-Raphson procedure, in which the stiffness matrix is updated at every equilibrium iteration, has been applied for solution. The importance of modifications of base functions for solving geometric nonlinear problems is analysed. The solved example is presented, the differences are compared and explained.
\end{abstract}

\section{Introduction}

Thin rectangular plates are the simplest shapes, easy to made therefore they are widely used in many branches of engineering. They occur as parts of aircraft, marine and vehicle structures in mechanical engineering, form bearing parts as well as reinforcements in civil engineering.

Thin plates subjected to the external pressure are liable to the buckling due to dominant compression membrane forces within the plate. Stored strain energy can be suddenly released and converted into kinetic energy with subsequent collapse of the structure. It is the reason why the stability problem has been analyzed since the beginning of the twenty century. Solving postbuckling behaviour of the compressed plate, nonlinear analysis is essential. It is also necessary to include initial imperfections of a real plate into the solution. Experience shows that small deviations from the prescribed geometry of a perfect plate may cause a strong reduction of the load-carrying capacity.

In this paper the effect of choice of base functions upon the mesh density of thin plate structures as well as upon the convergence of FEM solution of stability problems, have been studied. Numerical analysis of stability problems has been converging from above, therefore the mesh density should be identified at which the obtained results are applicable. Nonlinear solutions, to the contrary (incremental, refined by iterations) are time-consuming and the dense meshing is frequently inapplicable in practice. The paper is focused on a search for a proper type of a finite element. By sequential adding the degrees of freedom one can achieve higher accuracy using smaller number of iterations. Our aim is to find an optimal ratio between the speed of solution convergence and the degree of difficulty of

\footnotetext{
*Corresponding author: $\underline{\text { ivana.veghova@ stuba.sk }}$
} 
the calculation, to find the proper element for stability problems of thin plates, applicable also at small number of finite elements.

\section{Theoretical assumptions}

Geometrically nonlinear theory deals with more complicated strain - displacement equations. Let us assume a rectangular thin plate with the thickness $t$ simply supported along the edges (Fig. 1). The displacements of the point of the neutral surface are denoted $\mathbf{q}=\{\mathrm{u}, \mathrm{v}, \mathrm{w}\}^{\mathrm{T}}$ and the related load vector is denoted $\mathbf{p}=\left\{\mathrm{p}_{x}, 0,0\right\}^{\mathrm{T}}$. Assuming the so called von Kármán theory, the normal displacements (w) are much bigger than tangential displacements $(\mathrm{u}, \mathrm{v})$. By formulation of the strains, it has to be taken into account nonlinear terms.

Then $\boldsymbol{\varepsilon}=\boldsymbol{\varepsilon}_{l}+\boldsymbol{\varepsilon}_{n}-\boldsymbol{\varepsilon}_{b}$, where $\boldsymbol{\varepsilon}_{l}=\left\{\mathrm{u}_{, \mathrm{x}}, \mathrm{v}, \mathrm{y}, \mathrm{u}_{, \mathrm{y}}+\mathrm{v}_{, \mathrm{x}}\right\}^{\mathrm{T}}, \boldsymbol{\varepsilon}_{n}=\frac{1}{2}\left\{\mathrm{w}_{, \mathrm{x}}^{2}, \mathrm{w}_{, \mathrm{y}}^{2}, 2 \mathrm{w}_{, \mathrm{x}} \mathrm{w}_{, \mathrm{y}}\right\}^{\mathrm{T}}$, $\boldsymbol{\varepsilon}_{b}=\mathrm{z} \cdot \mathbf{k}=\mathrm{z} \cdot\left\{\mathrm{w}_{, \mathrm{xx}}, \mathrm{w}_{, \mathrm{yy}}, 2 \mathrm{w}_{, \mathrm{xy}}\right\}^{\mathrm{T}}$, the indexes denote the partial derivatives.

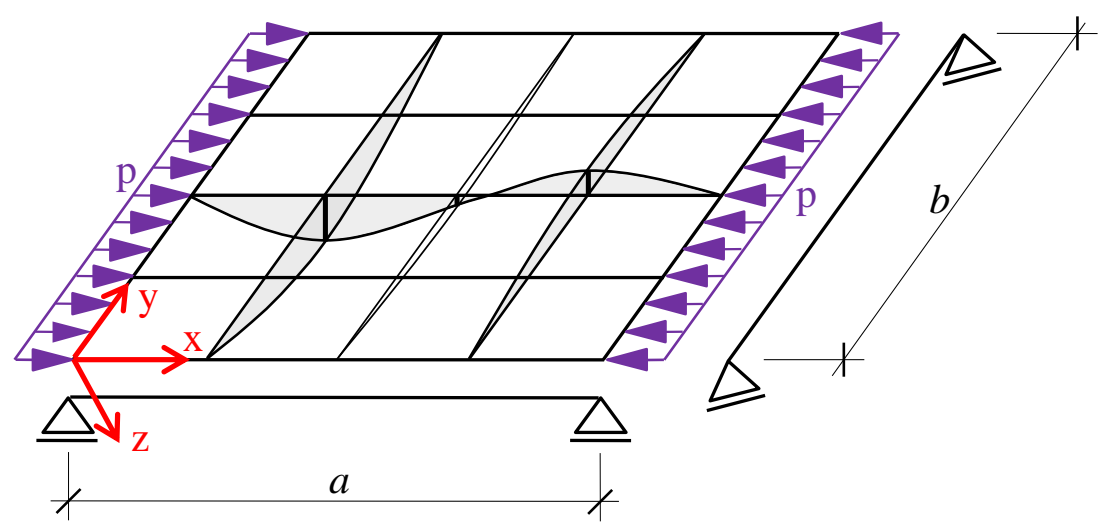

Fig. 1. Notations of the quantities of thin plate loaded in compression.

\subsection{Configuration of the System of Conditional Equations}

The increments of displacements of the point of neutral surface are denoted $\Delta \mathbf{q}=\{\Delta \mathrm{u}, \Delta \mathrm{v}, \Delta \mathrm{w}\}^{\mathrm{T}}$ and the increments of the strains can be written as:

$\Delta \boldsymbol{\varepsilon}=\left\{\begin{array}{l}\Delta \mathrm{u}_{, \mathrm{x}} \\ \Delta \mathrm{v}_{, \mathrm{y}} \\ \Delta \mathrm{u}_{, \mathrm{y}}+\Delta \mathrm{v}_{, \mathrm{x}}\end{array}\right\}+\left\{\begin{array}{l}\mathrm{w}_{, \mathrm{x}} \Delta \mathrm{w}_{, \mathrm{x}} \\ \mathrm{w}_{, \mathrm{y}} \Delta \mathrm{w}_{, \mathrm{y}} \\ \mathrm{w}_{, \mathrm{x}} \Delta \mathrm{w}_{, \mathrm{y}}+\mathrm{w}_{, \mathrm{y}} \Delta \mathrm{w}_{, \mathrm{x}}\end{array}\right\}+\frac{1}{2}\left\{\begin{array}{c}\Delta \mathrm{w}_{, \mathrm{x}}^{2} \\ \Delta \mathrm{w}_{, \mathrm{y}}^{2} \\ 2 \Delta \mathrm{w}_{, \mathrm{y}} \Delta \mathrm{w}_{, \mathrm{x}}\end{array}\right\}-\mathrm{z} \cdot\left\{\begin{array}{c}\Delta \mathrm{w}, \mathrm{xx} \\ \Delta \mathrm{w}_{, \mathrm{yy}} \\ 2 \Delta \mathrm{w}, \mathrm{xy}\end{array}\right\}$

Restricting to the isotropic elastic material and to the constant distribution of the residual stresses over the thickness, the increments of the stresses can be expressed as

$$
\Delta \boldsymbol{\sigma}=\left\{\begin{array}{l}
\Delta \sigma_{\mathrm{x}} \\
\Delta \sigma_{\mathrm{y}} \\
\Delta \tau
\end{array}\right\}=\mathbf{D} \Delta \boldsymbol{\varepsilon}, \text { where } \mathbf{D}=\frac{\mathrm{E}}{1-v^{2}}\left[\begin{array}{ccc}
1 & v & 0 \\
v & 1 & 0 \\
0 & 0 & \frac{1-v}{2}
\end{array}\right] \text { is the elasticity matrix. }
$$


The initial displacements will be assumed as the out of plane displacements only. For such defined task, one can express the increment of the total potential energy as:

$$
\begin{aligned}
& \Delta \mathrm{U}=\int_{V}\left(\frac{1}{2} \Delta \boldsymbol{\varepsilon}^{\mathrm{T}} \Delta \boldsymbol{\sigma}+\Delta \boldsymbol{\varepsilon}^{\mathrm{T}} \boldsymbol{\sigma}\right) d V-\int_{\Gamma} \Delta \mathbf{q}^{\mathrm{T}}(\mathbf{p}+\Delta \mathbf{p}) d \Gamma= \\
& =\int_{V}\left[\frac{1}{2} \Delta \boldsymbol{\varepsilon}^{\mathrm{T}} \mathbf{D} \Delta \boldsymbol{\varepsilon}+\Delta \boldsymbol{\varepsilon}^{\mathrm{T}} \mathbf{D}\left(\boldsymbol{\varepsilon}-\boldsymbol{\varepsilon}_{0}\right)\right] d V-\int_{\Gamma} \Delta \mathbf{q}^{\mathrm{T}}(\mathbf{p}+\Delta \mathbf{p}) d \Gamma
\end{aligned}
$$

The system of conditional equations one can get from the condition of the minimum of the increment of the total potential energy $\delta \Delta U=0$. After modifications this system can be written as:

$$
\mathbf{K}_{\text {inc }} \Delta \boldsymbol{\alpha}+\mathbf{F}_{\text {int }}-\mathbf{F}_{\text {ext }}-\Delta \mathbf{F}_{\text {ext }}=\mathbf{0},
$$

where $\mathbf{K}_{\text {inc }}=\left[\begin{array}{c:c}\mathbf{K}_{\text {incD }} & \mathbf{K}_{\text {incDS }} \\ \hdashline \mathbf{K}_{\text {incSD }} & \mathbf{K}_{\text {incS }}\end{array}\right]$ is the incremental stiffness matrix of the plate,

$\mathbf{F}_{\text {int }}=\left\{\begin{array}{c}\mathbf{F}_{\text {int } D} \\ \mathbf{F}_{\text {int } S}\end{array}\right\}, \mathbf{F}_{\text {ext }}=\left\{\begin{array}{c}\mathbf{F}_{\text {extD }} \\ \mathbf{F}_{\text {ext } S}\end{array}\right\}, \Delta \mathbf{F}_{\text {ext }}=\left\{\begin{array}{c}\Delta \mathbf{F}_{\text {extD }} \\ \Delta \mathbf{F}_{\text {ext }}\end{array}\right\}$ are the vector of the internal

forces, the vector of the external load and the vector of increments of the external load of the plate, respectively. More details can be found by the reader in [1].

\subsection{Solution of the system of conditional equations}

If we used a rectangular element obtained by composition of eight in-plane parameters and twelve plate parameters, we would have to choose high density of meshing to finite elements, in order to receive sufficiently suitable (accurate) solution. In other words, the solution would converge very slowly. In many papers [e.g. 2, 3] this phenomenon was demonstrated and illustrative examples are presented. Hence, for stability problems of thin plates was used a modified rectangular four-node finite element with 48 degrees of freedom (12 parameters in each node) with following choice of base functions [4].

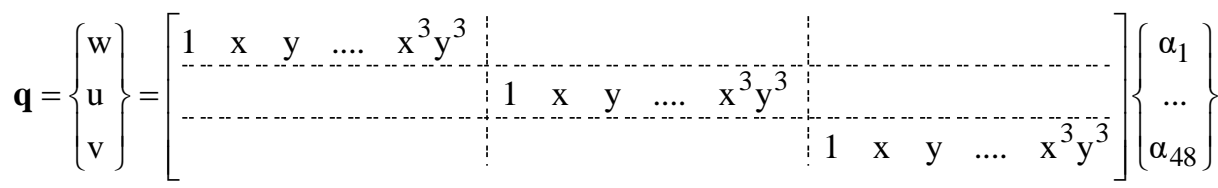

The solution of the system (5) cannot be achieved directly, and is based on a step by step incremental process that causes a deviation from the equilibrium nonlinear path. To correct this deviation, an iterative technique, Newton-Raphson method is used [5]. In the case of a more complicated loading path, it is possible to utilize a calculation of controlled displacement increment [6]. In the case of snap-back, this option fails, but there are still some perspectives in the possibility to change the pivot parameter during the calculation. In this case the interactive approach and calculation check are requisite.

\section{Design of computational model and numerical simulation}

Numerical analysis of steel $(\mathrm{E}=210 \mathrm{GPa}, v=0.3)$ rectangular plate $a=b=500 \mathrm{~mm}$, $t=4 \mathrm{~mm}$ is presented. Initial geometric imperfection coincided with the first buckling mode, with magnitude $0.1 \mathrm{~mm}$ is assumed. An example was chosen on purpose, in which the fundamental part of the loading path is monotone increasing. The calculation can be 
performed by classic NRM controlled by load increment. Constant value $\Delta \mathrm{p}=5 \mathrm{kN} / \mathrm{m}$ was considered for all types of finite elements and for each mesh density. The Ansys system [7] was used for analysis, these elements were stepwise used to create the model:

A) Shell 181 - classic bilinear element,

B) Shell 281 - 8 nodes biquadratic element with midside nodes,

C) User 33 - element with 48 D.O.F. according to (5) - aproximation with bicubic polynomial. In all cases the plate was modeled with $2 \times 2,4 \times 4,8 \times 8$ and $16 \times 16$ elements respectively (see Fig. 2).
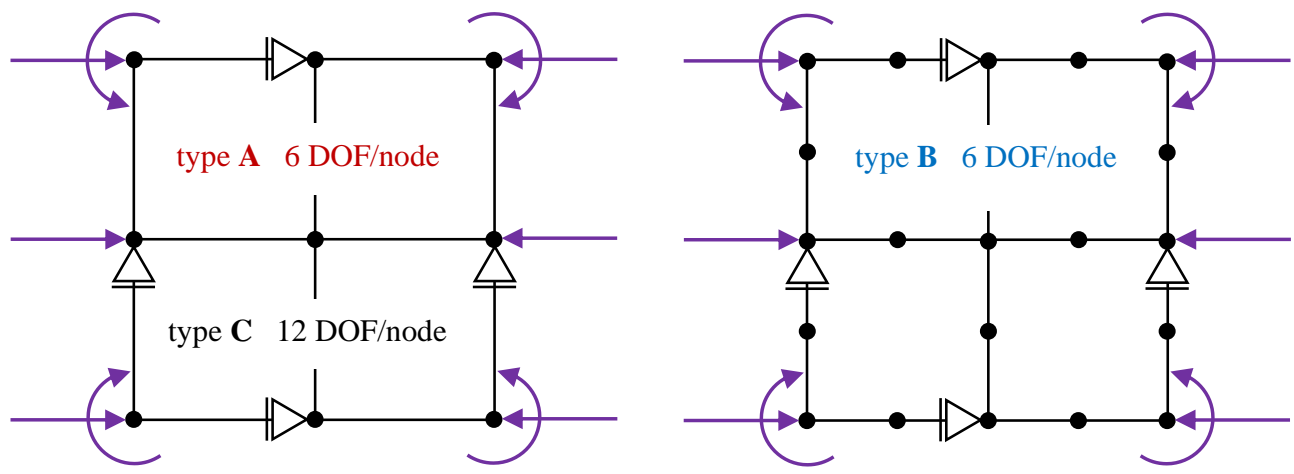

Fig. 2. FEM model - 2x2 elements. Shell 181 resp. User 33 on the left, Shell 281 on the right.

The calculation with the prescribed load increment was running up to the load level of $400 \mathrm{kN} / \mathrm{m}$, containing the post-buckling phase of loading (the critical load value for the perfect plate $\mathrm{q}_{\text {crit }}=194.36 \mathrm{kN} / \mathrm{m}$ ).

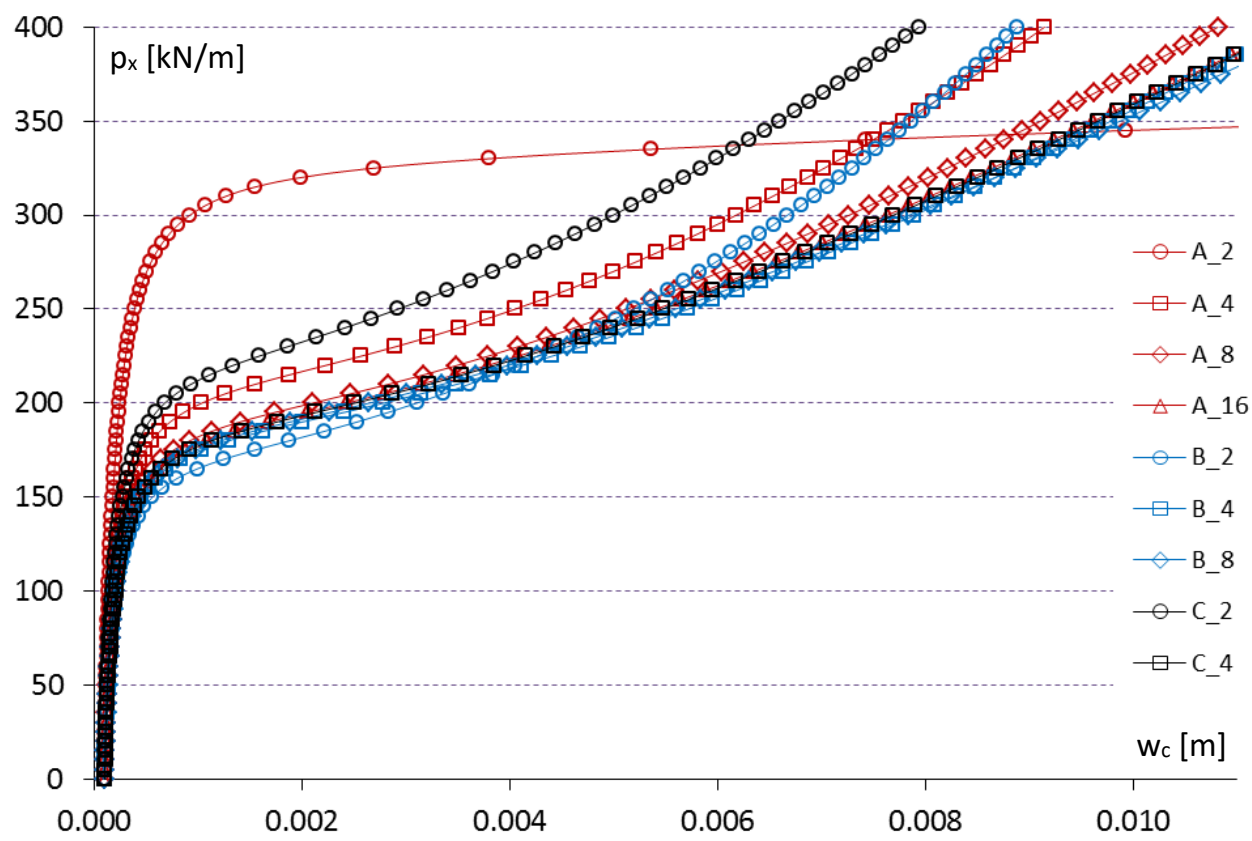

Fig. 3. The equilibrium path for the central node $\mathrm{w}_{\mathrm{c}}(a / 2, b / 2)$. 
Obtained results have been arranged into equilibrium paths for central node $(a / 2, b / 2)$ in Fig. 3. For element of type A (Shell 181) solutions are plotted in red colour, systematically for different mesh densities. It has been processed in the same way and rendered in blue the type B (Shell 281) and rendered in black the type C (User 33). In Fig. 4 only a portion of the equilibrium paths in postbuckling phase $(200 \mathrm{až} 250 \mathrm{kN} / \mathrm{m})$ is presented. One can see that paths representing solutions for A 16, B 8 and $\mathbf{C} 4$ are located close to each other. This means, that from the viewpoint of the accuracy of the solution it has been comparable to create thin plate using 16x16 elemens of the type Shell 181 (bilinear), $8 \times 8$ elemens of the type Shell 281 (biquadratic) and 4x4 elemens of the type User 33 (bicubic).

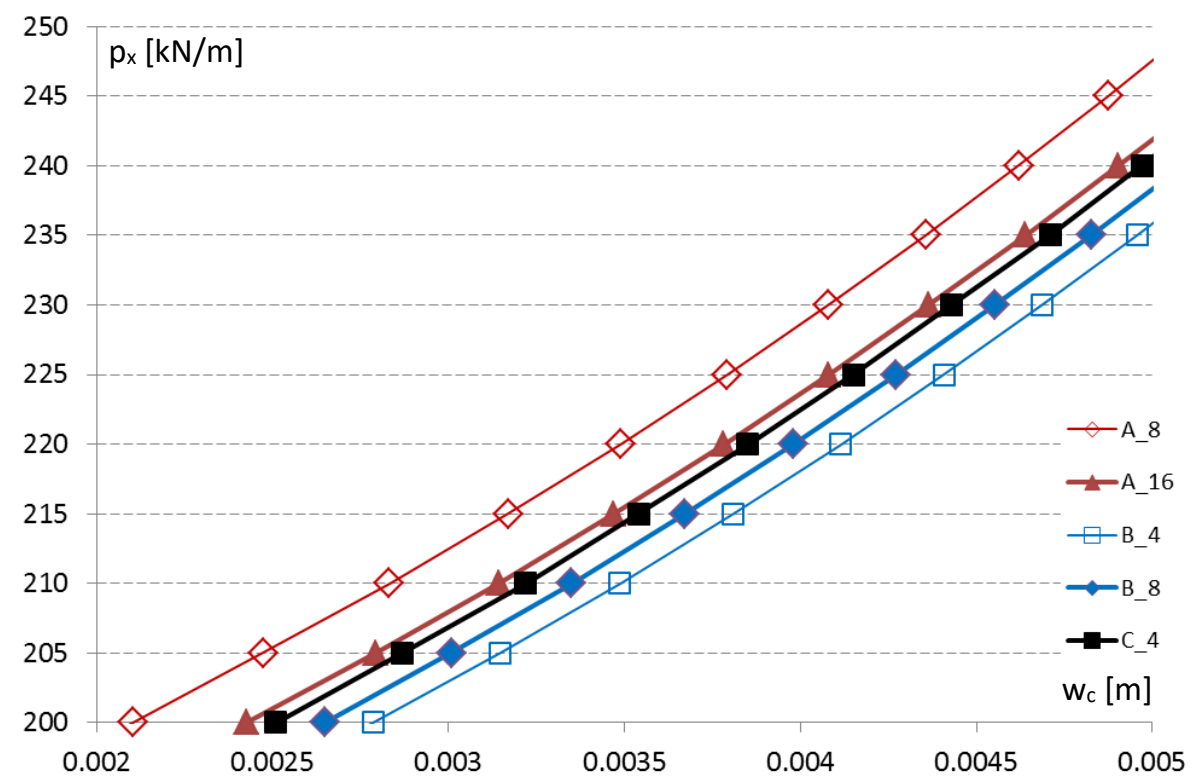

Fig. 4. Detail of the equilibrium path, postbuckling phase $(200-250 \mathrm{kN} / \mathrm{m})$.

Since the three options mentioned appear to be, from the viewpoint of the convergence to an exact solution, comparable in their benefits, let us consider the second criterion, i.e. the complexity of the calculation. For this we can use Table 1 that contains all results compiled in a clear manner.

Table 1. The complexity of the calculation.

\begin{tabular}{|c|c|c|c|c|c|}
\hline & Elements & Nodes & Nodal par. & No. eq. & (No. steps) \\
\hline A 2 & 4 & 9 & 54 & 42 & nonconverg. \\
A 4 & 16 & 25 & 150 & 130 & 80 \\
A 8 & 64 & 81 & 486 & 450 & 80 \\
A 16 & $\mathbf{2 5 6}$ & $\mathbf{2 8 9}$ & $\mathbf{1 7 3 4}$ & $\mathbf{1 6 6 6}$ & 80 \\
B 2 & 4 & 21 & 126 & 106 & 80 \\
B 4 & 16 & 65 & 390 & 354 & 80 \\
B 8 & $\mathbf{6 4}$ & $\mathbf{2 2 5}$ & $\mathbf{1 3 5 0}$ & $\mathbf{1 2 8 2}$ & 80 \\
C 2 & 4 & 9 & 108 & 84 & 80 \\
C 4 & $\mathbf{1 6}$ & $\mathbf{2 5}$ & $\mathbf{3 0 0}$ & $\mathbf{2 6 0}$ & 80 \\
\hline
\end{tabular}




\section{Conclusions}

If we compare a number of equations that must be created and solved in each iteration (which also represents a dimension of matrix $\mathrm{K}_{\text {inc, }}$, which has to be repeatedly factorized) we realize that: while in cases $\mathbf{A}$ and $\mathbf{B}$ the numbers are comparable, dimension $\mathbf{C}$ is smaller and the solution is faster. Utilizing of this element in the solution therefore appears to be more favorable. To formulate a more general conclusion, one can say that making the solution more accurate by increasing the number of nodes in element is not a very appropriate option. Increasing of the number of nodal parameters seems to be preferable for this purpose.

Presented results have been arranged due to the research supported by the Slovak Scientific Grant Agency, project No. 1/0265/16.

\section{References}

1. M. Psotny, J. Ravinger, Engineering Mechanics, 14 (2007)

2. M. A. Crisfield, Non-Linear Finite Element Analysis of Solids and Structures (Wiley\&Sons, London, 1996)

3. K. J. Bathe, Finite element procedures in engineering analysis (Prentice-Hall, Englewood Cliffs, 1982)

4. S. Saigal, I. Yang, Int. J. Numer. Methods in Engng., 22 (1985)

5. D. W. Murray, E. L. Wilson, AIAA J., 7 (1969)

6. J. L. Batoz, G. Dhatt, Int. J. Numer. Meth. Eng., 14 (1979)

7. ANSYS User's Manual 13.0. Swanson Analysis Systems, Inc., (2010) 